\title{
ASSESSMENT OF EXTRAPULMONARY TOXICITY INDUCED BY CARBON NANOMATERIALS FOLLOWING INTRATRACHEAL INSTILLATION IN RATS
}

\author{
BHIKKU ANGOTH, HARIKIRAN LINGABATHULA, NARSIMHAREDDY YELLU* \\ Department of Pharmacology and Toxicology, University College of Pharmaceutical Sciences, Kakatiya University, Warangal, \\ Telangana, India. Email: ynrkuc@gmail.com
}

Received: 18 January 2016, Revised and Accepted: 01 February 2017

\section{ABSTRACT}

Objective: Carbon nanomaterials (CNMs) such as carbon nanofibres (CNFs), multi-wall carbon nanotubes (MWCNTs), and carbon nanorods (CNRs) were found various industrial and commercial applications. The occupational exposure for these CNMs was also increased enormously. This study evaluated the extrapulmonary toxicity induced by these CNMs.

Methods: The extrapulmonary toxicity was assessed following intratracheal instillation of test CNMs in rats after 1 day, 1 week, 1 month, and 3 months postexposure periods using serum biochemical parameters such as alanine transaminase (ALT) and creatinine using diagnostic assay kits. Further, the histopathological analysis was performed for liver and kidneys of particle exposed rats.

Results: The results have displayed that increased levels of serum ALT and creatinine were found after 1 day, 1 week, and 1 month postexposure periods indicating liver and kidney toxicity, respectively. This toxicity was further confirmed by the changes observed in the histopathological analysis of rat liver and kidneys.

Conclusion: The CNFs, MWCNTs, and CNRs able to translocate from the lungs into other extrapulmonary organs such as liver and kidney, and also cause dose-dependent toxicity to them.

Keywords: Alanine transaminase, Carbon nanomaterials, Creatinine, Toxicity.

(C) 2017 The Authors. Published by Innovare Academic Sciences Pvt Ltd. This is an open access article under the CC BY license (http://creativecommons. org/licenses/by/4. 0/) DOI: http://dx.doi.org/10.22159/ajpcr.2017.v10i5.17152

\section{INTRODUCTION}

Carbon nanomaterials (CNMs) are a new form of crystalline carbon currently attracting intense research efforts because of their unique properties that make them suitable, as such or after modification, for many industrial developments such as in high strength materials, electronics or biomedical applications [1]. With the widespread development of commercial CNMs manufacturing and commercial application, CNMs such as multi-wall carbon nanotubes (MWCNTs), carbon nanofibers (CNF), and carbon nanorods (CNRs) are an important category of nanoparticle for health risk assessment. There is a need to address the associated bioactivity of these newly manufactured nanomaterials $[2,3]$.

Pulmonary retention and extrapulmonary redistribution of inhaled nanoparticles have been considered to be important contributing factors of cardiorespiratory diseases. Varying the characteristics of carbon nanoparticles, such as size, surface charge, attachment of ligands, or surfactant coatings, offers the possibility for site-specific targeting of different regions of the gastro-intestinal tract. The fast transit of material through the intestinal tract (on the order of hours), together with the continuous renewal of epithelium, led to the hypothesis that nanomaterials will not remain there for indefinite periods [4].

The ingested nanoparticles have eliminated rapidly: $98 \%$ in the feces within 48 hours and most of the remainder via urine [3]. However, other studies indicate that certain nanoparticles can translocate to blood, spleen, liver, bone marrow, lymph nodes, kidneys, lungs, brain and can also be found in the stomach and small intestine. Oral uptake of polystyrene spheres of various sizes $(50 \mathrm{~nm}-3 \mu \mathrm{m})$ by rats resulted in a systemic distribution to liver, spleen, blood, and bone marrow [5].
Recently, some investigations were evaluated the in vitro toxicity of different nanoparticles in human cell lines through various biomarkers and reported the cytotoxicity and oxidative stress induction [6-8]. Initial studies focused on the respiratory effects of pulmonary exposure of various nanoparticles but were limited by the lack of knowledge concerning the extra-pulmonary toxicity [9-11]. Some nanoparticles characterized by their ability to translocate, from their site of deposition in the lungs to the blood and the brain $[12,13]$. In this study, we have evaluated the ability of the MWCNTs, CNFs, and CNRs to translocate into extra-pulmonary organs like liver and kidney, and also evaluated the extrapulmonary toxicity of these CNMs in rats following intratracheal instillation.

\section{METHODS}

The MWCNTs, CNFs, and CNRs were purchased from Sigma-Aldrich, USA. Quartz powder (QZ) and carbonyl iron (CI) were obtained from S.D. Fine chemicals, India. Phosphate buffer saline (PBS) and Tween 80 were procured from HiMedia Laboratories Ltd., Mumbai. Serum alanine transaminase (ALT) and serum creatinine assay kits were purchased from Span diagnostics, India.

\section{Experimental animals}

Groups of male wistar albino rats (Hyderabad, India) of 6-9 weeks old at study start (mean weights in the range of 220-275 g) were selected and housed in polypropylene cages in a room where the congenial temperature was $26 \pm 2^{\circ} \mathrm{C}$ and $12 \mathrm{hrs}$ light and dark cycles were maintained. The animals were allowed to acclimatize to the environment for 7 days and supplied with a standard pellet diet and water ad libitum. All procedures using animals were reviewed and approved by the Institutional Animal Care and Use Committee of Kakatiya University. 
Preparation of fine-dust suspensions

Fine particle suspensions of CNMs were prepared with a nontoxic dispersion vehicle for instillation into rat lungs [14,15]. The products are extremely difficult to disperse even in the presence of a dispersing agent. All the CNMs suspensions were prepared in PBS $+1 \%$ Tween 80 at a concentration of $10 \mathrm{mg} / \mathrm{mL}$ by briefly shearing ( 2 minutes in a small glass homogenizing tube) and subsequently sonicating (1-2 minutes) the samples. All the samples with different concentrations were re-sonicated on the day of dosing before the instillation.

\section{Intratracheal instillation}

The rats were anesthetized with $3-5 \%$ isoflurane in a small chamber and individual rats were secured on an inclined plastic platform and anesthetization continued via a small nose cone. The trachea was exposed by a $1 \mathrm{~cm}$ incision on the ventral neck skin for instillation of the dust suspension [16]. The intratracheal fast instillation/nebulization procedure for rats was modified to ensure that instilled material was delivered into the lungs of rats with a good distribution [17].

\section{Experimental design}

Groups of rats were instilled intratracheally with single dose of $1 \mathrm{mg} / \mathrm{kg}$ or $5 \mathrm{mg} / \mathrm{kg}$ of CNF, MWCNT, CNR, CI and quartz-crystalline silica particles (QZ). All the particles were prepared in a volume of $1.0 \%$ Tween 80 and phosphate-buffered saline and subjected to polytron dispersion [18]. Groups of PBS-Tween, CI, and QZ instilled rats were served as solvent control, negative control, and positive control, respectively.
Collection of blood samples and biochemical analysis

Blood samples were collected from all the group of rats by retroorbital sinus puncture, under mild ether anesthesia and serum was obtained by immediate centrifugation of blood samples using BIOFUGE cooling centrifuge at $3000 \mathrm{rpm}$ for 10 minutes at room temperature. The blood samples were collected from all the group of rats at $24 \mathrm{hrs}, 1$ week, 1 month, and 3 months postinstillation intervals and were used to estimate suitable biomarkers (enzyme levels) which represent the organ/tissue toxicity. Evaluation of ALT levels was specifically used to assess the liver toxicity and estimation of serum creatinine levels assesses the renal toxicity. The biochemical assays were performed on serum samples for the estimation of creatinine and ALT using respective diagnostic kit methods.

\section{Histopathological studies of extrapulmonary organs}

The extrapulmonary vital organs (liver and kidney) of all the control and particles exposed rats were collected at $24 \mathrm{hrs}, 1$ week, 1 month and 3 months postinstillation intervals of CNMs. These tissues were used for the histopathological examination to assess the extrapulmonary toxicity of CNMs. The isolated tissues were fixed in $10 \% \mathrm{v} / \mathrm{v}$ neutral buffered formalin and processed using routine histological techniques. The liver and kidney tissues were embedded in paraffin and stained using hematoxylin and eosin ( $\mathrm{H}$ and $\mathrm{E}$ ) dyes for histopathological evaluations.

\section{Statistical analysis}

All the experimental values were expressed as mean \pm standard deviation and were compared with control value at each time point. One-way analysis of variance and Dunnett test were used to compare means

Table 1: Serum ALT levels (IU/L) in rats exposed to CNFs, MWCNTs and CNRs

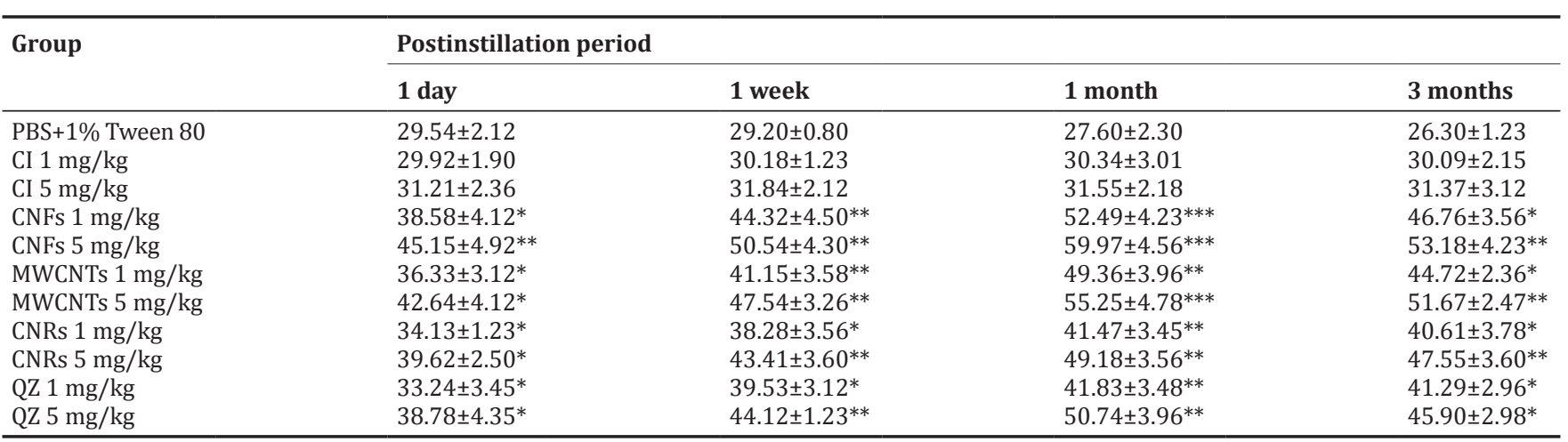

Data were expressed as mean \pm SD $(n=6)$, Significance was indicated by ${ }^{*} \mathrm{p}<0.05,{ }^{* *} \mathrm{p}<0.01,{ }^{* * *} \mathrm{p}<0.001$ versus control treated rats. ALT: Alanine transaminase,

BPS: Phosphate buffer saline, CI: Carbonyl iron, CNFs: Carbon nanofibers, MWCNTs: Multi wall carbon nanotubes, CNRs: Carbon nanorods, QZ: Quartz, SD: Standard deviation

Table 2: Serum creatinine levels (mg/dL) in rats exposed to CNFs, MWCNTs and CNRs

\begin{tabular}{|c|c|c|c|c|}
\hline \multirow[t]{2}{*}{ Group } & \multicolumn{4}{|c|}{ Postinstillation period } \\
\hline & 1 day & 1 week & 1 month & 3 months \\
\hline PBS+1\% Tween 80 & $0.79 \pm 0.11$ & $0.79 \pm 0.10$ & $0.75 \pm 0.23$ & $0.72 \pm 0.20$ \\
\hline CI 5 mg/kg & $0.82 \pm 1.12$ & $0.82 \pm 0.18$ & $0.91 \pm 0.18$ & $0.84 \pm 0.16$ \\
\hline CNFs $1 \mathrm{mg} / \mathrm{kg}$ & $1.04 \pm 0.25^{*}$ & $1.08 \pm 0.32 *$ & $1.29 \pm 0.32^{* *}$ & $1.03 \pm 0.27$ \\
\hline CNFs $5 \mathrm{mg} / \mathrm{kg}$ & $1.29 \pm 0.28^{* *}$ & $1.41 \pm 0.39 * * *$ & $1.32 \pm 0.42^{* *}$ & $1.12 \pm 0.35$ \\
\hline MWCNTs $1 \mathrm{mg} / \mathrm{kg}$ & $1.02 \pm 0.16^{*}$ & $1.06 \pm 0.25^{*}$ & $1.23 \pm 0.32 *$ & $1.02 \pm 0.29$ \\
\hline MWCNTs $5 \mathrm{mg} / \mathrm{kg}$ & $1.26 \pm 0.25^{* *}$ & $1.36 \pm 0.40^{* *}$ & $1.25 \pm 0.41^{* *}$ & $1.08 \pm 0.16$ \\
\hline CNRs $1 \mathrm{mg} / \mathrm{kg}$ & $0.98 \pm 0.26$ & $1.01 \pm 0.34^{*}$ & $1.12 \pm 0.34^{*}$ & $1.04 \pm 0.34$ \\
\hline CNRs $5 \mathrm{mg} / \mathrm{kg}$ & $1.23 \pm 0.31^{*}$ & $1.31 \pm 0.23^{* *}$ & $1.23 \pm 0.36^{*}$ & $1.04 \pm 0.23$ \\
\hline QZ $1 \mathrm{mg} / \mathrm{kg}$ & $0.92 \pm 0.19$ & $0.98 \pm 0.19$ & $1.11 \pm 0.45^{*}$ & $1.07 \pm 0.35$ \\
\hline QZ $5 \mathrm{mg} / \mathrm{kg}$ & $1.22 \pm 0.23^{*}$ & $1.29 \pm 0.36^{* *}$ & $1.21 \pm 0.39 *$ & $1.03 \pm 0.26$ \\
\hline
\end{tabular}

Data were expressed as mean $\pm \mathrm{SD}(\mathrm{n}=6)$; Significance was indicated by ${ }^{*} \mathrm{p}<0.05,{ }^{* *} \mathrm{p}<0.01,{ }^{* * *} \mathrm{p}<0.001$ versus control treated rats. BPS: Phosphate buffer saline,

CI: Carbonyl iron, CNFs: Carbon nanofibers, MWCNTs: Multi wall carbon nanotubes, CNRs: Carbon nanorods, QZ: Quartz, SD: Standard deviation 
from the control group and each of the groups exposed to particulates, and the statistical significance was judged at the 0.05 probability level. Significance was indicated by: ${ }^{*} \mathrm{p}<0.05,{ }^{* *} \mathrm{p}<0.01,{ }^{* * *} \mathrm{p}<0.001$ versus control $(n=6)$

\section{RESULTS}

Similar to the QZ, exposures of CNFs, MWCNTs, and CNRs in rats resulted in a dose-dependent increase in ALT levels at all postexposure periods (Table 1), but the significant $(\mathrm{p}<0.001)$ increase in ALT levels were observed with $5 \mathrm{mg} / \mathrm{kg}$ doses of all CNMs.
Intratracheal instillation of CNFs, MWCNTs, and CNRs in rats resulted in a dose-dependent increase in creatinine levels at all postexposure periods (Table 2), but the significant $(\mathrm{p}<0.001)$ increase in creatinine levels were observed with $5 \mathrm{mg} / \mathrm{kg}$ dose at 1 week of postinstillation period of CNFs.

The liver and kidney tissues exposed to various CNMs were isolated at 1 day, 1 week, 1 month, and 3 months postexposure periods and the histopathological changes were presented in Figs. 1 and 4. The rat livers after 1 week and 1 month postinstillation periods have shown congestion of central vein, shrinkage or ballooming of

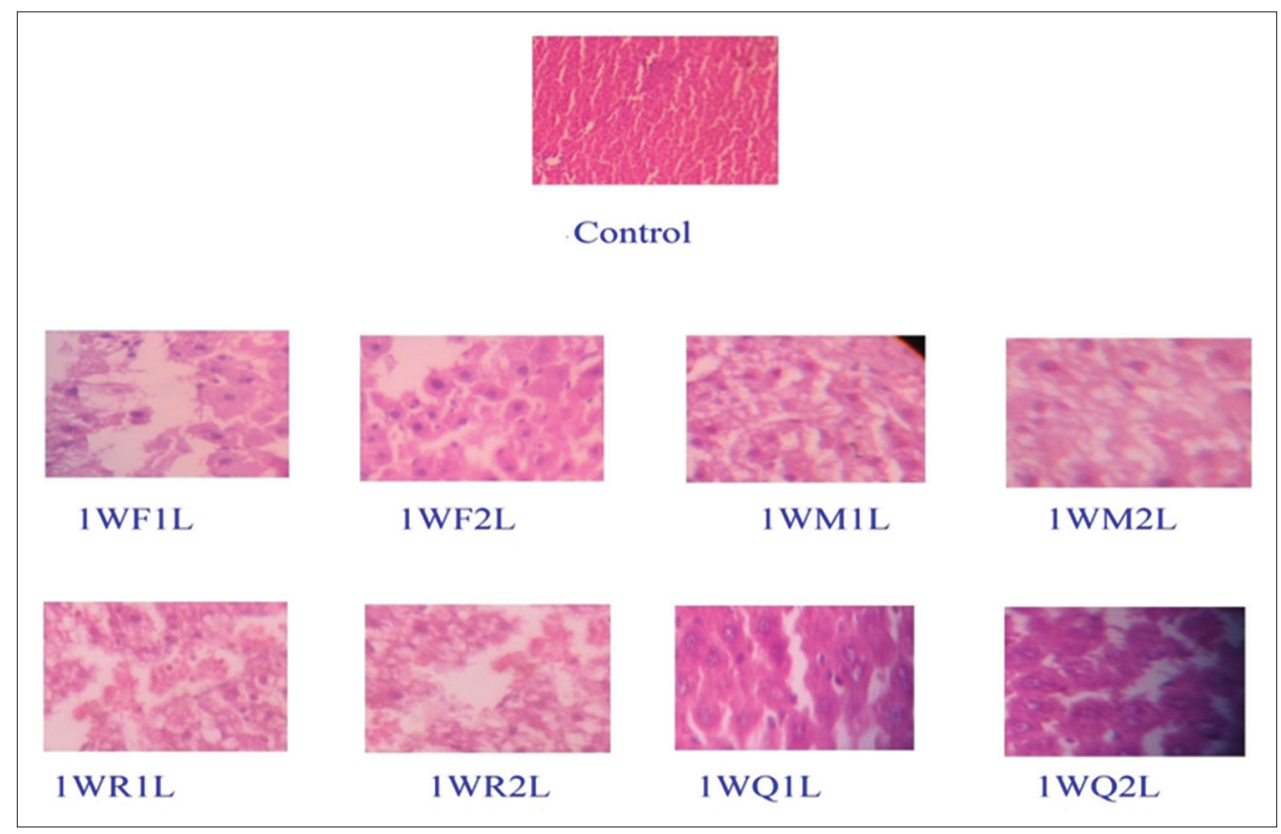

Fig. 1: Light micrograph of rat liver tissue at 1 week postinstillation exposure; control: PBS+1\% Tween 80; 1WF1L: CNF (1 mg/kg); 1WF2L: CNF (5 mg/kg); 1WM1L: MWCNT (1 mg/kg); 1WM2L: MWCNT (5 mg/kg); 1WR1L: CNR (1 mg/kg); 1WR2L: CNR (5 mg/kg); 1WQ1L: Quartz $(1 \mathrm{mg} / \mathrm{kg})$; 1WQ2L: Quartz (5 mg/kg)

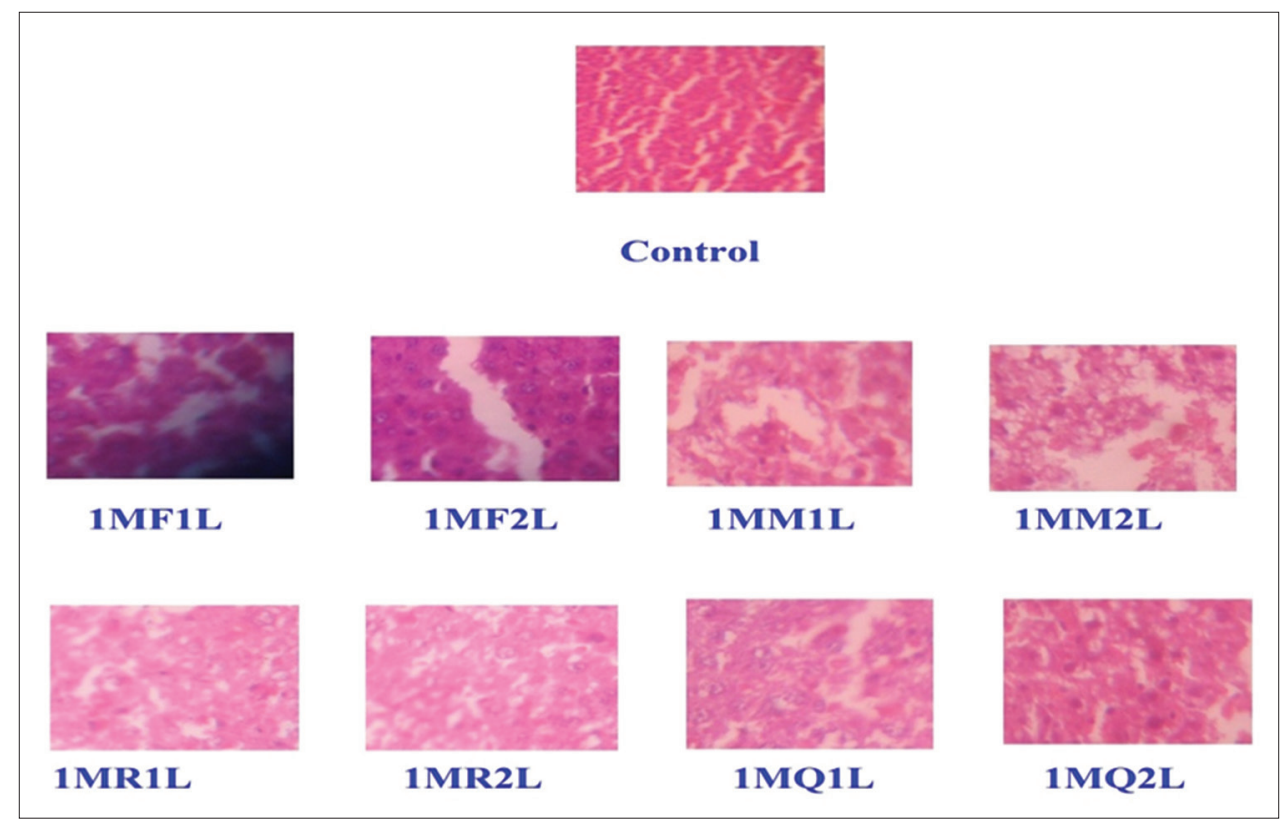

Fig. 2: Light micrograph of rat liver tissue at 1 month postinstillation exposure; control: PBS+1\% Tween 80; $1 \mathrm{MF} 1 \mathrm{~L}$ : CNF (1 mg/kg); 1MF2L: CNF (5 mg/kg); 1MM1L: MWCNT (1 mg/kg); 1MM2L: MWCNT (5 mg/kg); 1MR1L: CNR (1 mg/kg); 1MR2L: CNR (5 mg/kg) 1MQ1L: Quartz (1mg/kg); 1MQ2L: Quartz (5 mg/kg) 


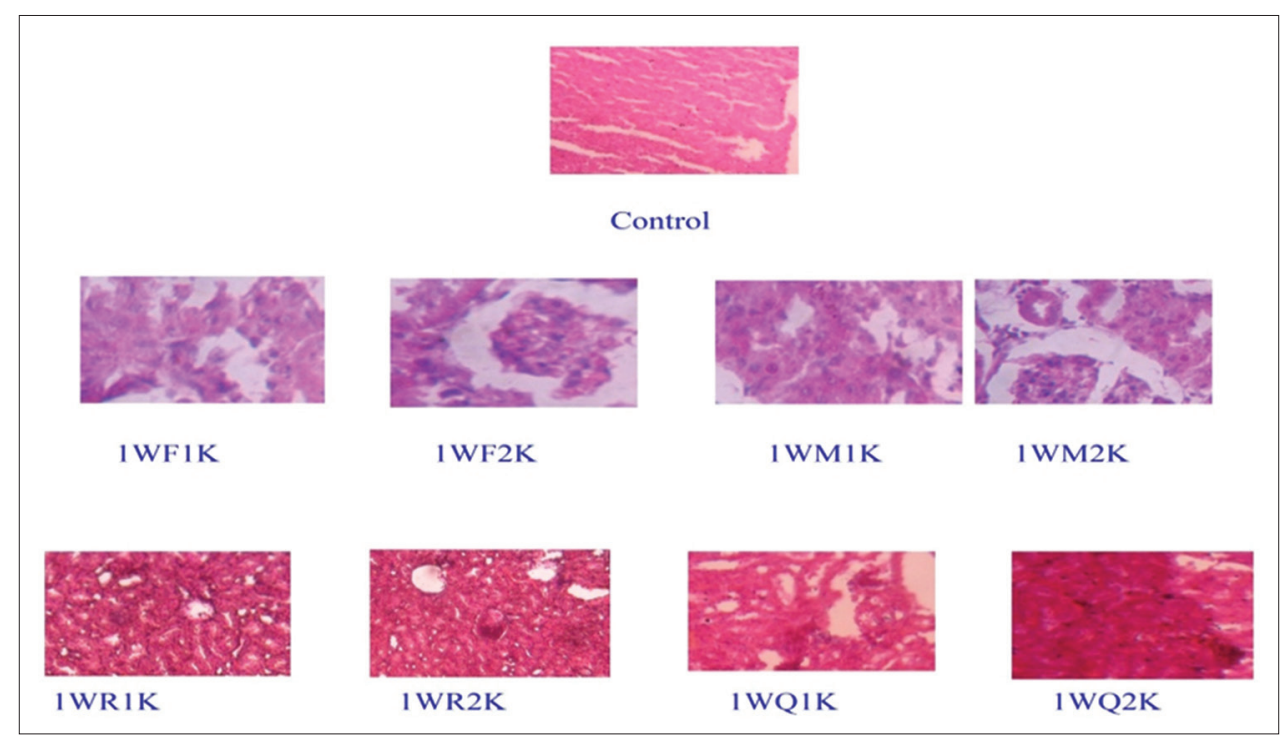

Fig. 3: Light micrograph of rat kidney tissue at 1 week postinstillation exposure; control: PBS+1\% Tween 80; $1 \mathrm{WF} 1 \mathrm{~K}$ : CNF (1 mg/kg), 1WF2K: CNF (5 mg/kg) WM1K: MWCNT (1 mg/kg); 1WM2K: MWCNT (5 mg/kg); 1WR1K: CNR (1 mg/kg); 1WR2K: CNR (5 mg/kg); 1WQ1K: Quartz (1 mg/kg); 1WQ2K: Quartz (5 mg/kg)

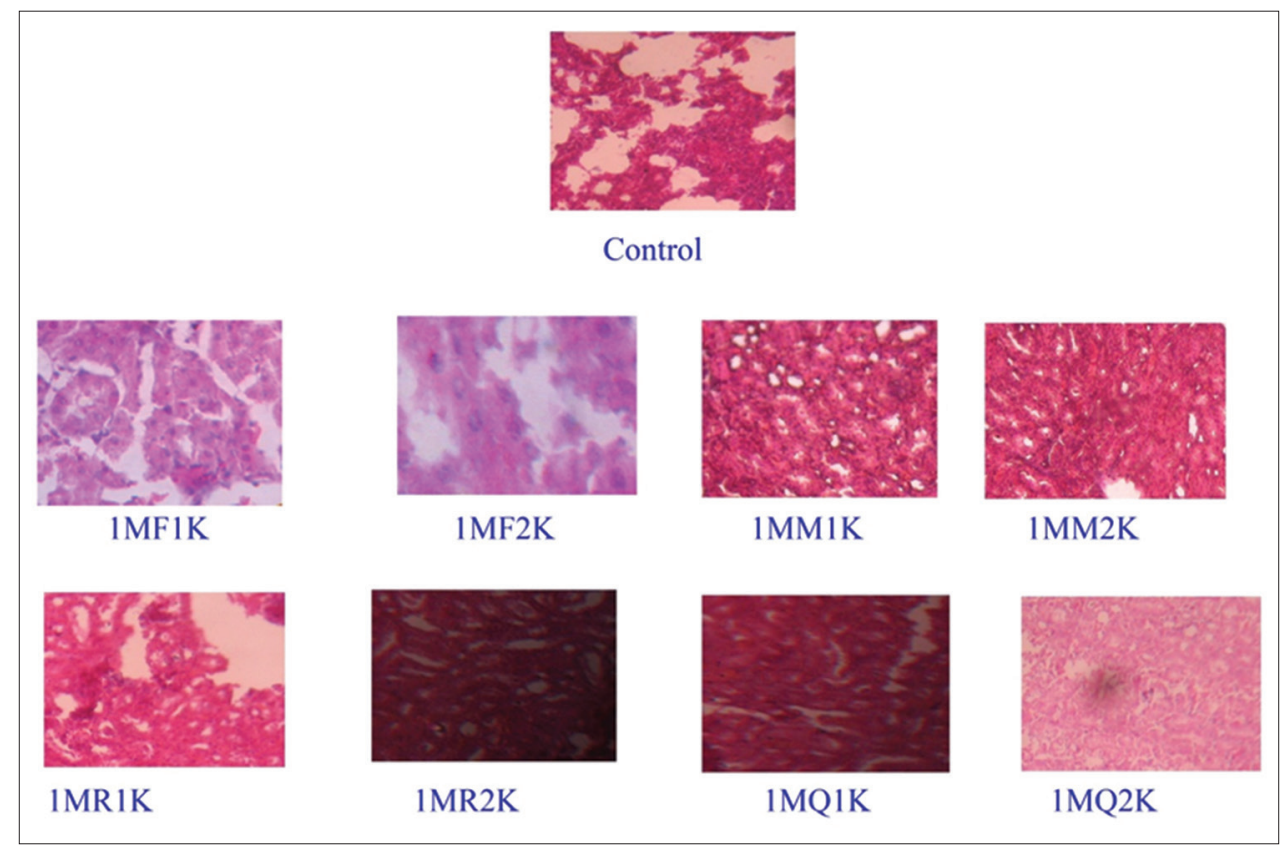

Fig. 4: Light micrograph of rat kidney tissue at 1 month postinstillation exposure; control: PBS+1\% Tween 80; 1MF1K: CNF (1 mg/kg); 1MF2K: CNF (5 mg/kg); 1MM1K: MWCNT (1 mg/kg); 1MM2K: MWCNT (5 mg/kg); 1MR1K: CNR (1 mg/kg); 1MR2K: CNR (5 mg/kg); 1MQ1K: Quartz (1 mg/kg); 1MQ2K: Quartz (5 mg/kg)

hepatocytes, necrosis, and degeneration with fat globules. There were no remarkable changes in liver tissue during 1 day and 3 months postexposure periods.

The histology of rat kidneys after 1 week and 1 month postexposure was displayed tubular dilation, increased inflammatory infiltrates, thinned outer cortex, focal areas showing necrosis and degeneration. There were no remarkable changes were found in the kidneys after 1 day and 3 months postexposure periods.

\section{DISCUSSION}

This study investigated the ability of the different types of carbon nanoparticles to translocate into extrapulmonary organs such as liver and kidney, and also evaluated the extrapulmonary toxicity in rats following intratracheal instillation of CNMs. Exposure of these CNMs at different doses does not produce any mortality in exposed rats. Exposure of QZ and these CNMs produced a dose-dependent periportal lymphocytic infiltration, congestion of sinusoids, ballooning, foamy degeneration of hepatocytes, fatty changes and finally focal inflammation and necrosis at 1 week and 1 month postinstillation periods. However, the significant tubular necrosis and interstitial nephritis take place with a dose of $5 \mathrm{mg} / \mathrm{kg}$ at 1 week of postinstillation of CNFs.

Exposures of these CNMs produced progressive liver and kidney damage which were confirmed by elevated levels of respective 
biomarkers of cell injury and inflammation. Analysis of serum for tissue damage biomarkers (serum ALT and creatinine) levels revealed that these enzyme levels in CNMs exposed rats ( 1 and $5 \mathrm{mg} / \mathrm{kg} \mathrm{b.w}$ ) were significantly higher than control at all the mentioned postexposure periods. The results of this study also showed the greater toxicity of CNFs than MWCNTs and CNRs.

Some of the reported the pulmonary toxicities induced by different CNMs such as single wall carbon nanotubes, MWCNTs and CNFs following intratracheal instillation in rats after 1 day, 1 week, 1 month, and 3 months postexposure periods [19-21]. However, our results suggest the dose-dependent extra-pulmonary (liver and kidney) toxicities of intratracheally instilled CNFs, MWCNTs, and CNRs in rats, which was further confirmed by the histopathological examination of these organs of particle exposed rats. These extrapulmonary toxicities of CNMs might be due to the translocation into the liver and kidneys.

Once the particles have reached pulmonary interstitial sites, uptake into the blood circulation in addition to lymphatic pathways can occur, a pathway that again is dependent on particle size, favoring nanosized particles. Translocation of nanoparticles from the lung to the blood and extrapulmonary organs has been documented for various nanoparticles. For example, 60 minutes after intratracheal instillation of hamsters with nanocolloid albumin labeled with technetium-99m, detectable levels of the labeled nanoparticles were found in the blood, liver, heart, spleen, kidneys, and brain [13]. In ththis study also, MWCNT translocate mainly into the liver and produces liver toxicity than other extra-pulmonary sites.

Berry et al. was the first to describe translocation of nano sized particles across the alveolar epithelium using intratracheal instillations of $30 \mathrm{~nm}$ gold particles in rats [22]. Evidence in humans for the translocation of inhaled NSP into the blood circulation is ambiguous, with one study showing rapid appearance in the blood and significant accumulation of label in the liver of humans inhaling 99Tc-labelled $20 \mathrm{~nm}$ carbon particles [12], while another study using the same labeled particles reported no such accumulation [23].

Taken together all of the evidence from animal and human studies for alveolar translocation of various nanoparticles, it is likely that this pathway exists in humans as well; however, in addition to particle size, the extent of extrapulmonary translocation is highly dependent on particle surface chemistry, the amount of inhaled nanoparticles and potential degradation by lysosomal enzymes before transport to the lymphatic circulation. Translocation to the blood circulation could provide a mechanism for a direct particle effect on the cardiovascular system as an explanation for epidemiological findings of cardiovascular effects associated with inhaled ambient ultra fine particles [24-26]. All these above studies were supported our results for translocation and extrapulmonary toxicity induced by the test CNMs.

\section{CONCLUSION}

In summary, the intratracheal instillation of test CNMs increased the serum ALT and creatinine levels following 1 day, 1 week and 1 month postexposure periods resulting in liver and kidney toxicity, respectively. This further confirmed by histopathological analysis of liver and kidneys. The order of extrapulmonary toxicity induced by the test CNMs was CNFs > MWCNTs > CNRs. Finally, the CNFs, MWCNTs, and CNRs able to translocate from the lungs into other extrapulmonary organs such as liver and kidney, and also cause dose-dependent toxicity to them.

\section{REFERENCES}

1. Prasanta D, Nilimanka D. Carbon nanotubes: It's role in modern health care. Int J Pharm Pharm Sci 2013;5(4):9-13.

2. Oberdörster E. Manufactured nanomaterials (fullerenes, C60) induce oxidative stress in the brain of juvenile largemouth bass. Environ Health Perspect 2004;112(10):1058-62.

3. Oberdörster G, Oberdörster E, Oberdörster J. Nanotoxicology: An emerging discipline evolving from studies of ultrafine particles. Environ Health Perspect 2005;113(7):823-39.

4. Hoet PH, Brüske-Hohlfeld I, Salata OV. Nanoparticles - Known and unknown health risks. J Nanobiotechnology 2004;2(1):12.

5. Deloncle R, Guillard O, Huguet F, Clanet F. Modification of the bloodbrain barrier through chronic intoxication by aluminum glutamate. Possible role in the etiology of Alzheimer's disease. Biol Trace Elem Res 1995;47(1-3):227-33.

6. Lingabathula $\mathrm{H}$, Yellu N. Cytotoxicity, oxidative stress, and inflammation in human Hep G2 liver epithelial cells following exposure to gold nanorods. Toxicol Mech Methods 2016;26(5):340-7.

7. Harikiran L, Bhikku A, Narsimha RY. In vitro cytotoxicity of gold and silver nanorods using different human cell lines. Lat Am J Pharm 2015;34(7):1277-82

8. Bhikku A, Harikiran L, Durgaiah G, Narsimha RY. Cytotoxicity evaluation of carbon nanomaterials on human cell lines using MTT assay. Int J Pharm Pharm Sci 2014;6(10):379-82.

9. Gelli K, Porika M, Anreddy RN. Assessment of pulmonary toxicity of $\mathrm{MgO}$ nanoparticles in rats. Environ Toxicol 2015;30(3):308-14.

10. Warheit DB, Brock WJ, Lee KP, Webb TR, Reed KL. Comparative pulmonary toxicity inhalation and instillation studies with different $\mathrm{TiO} 2$ particle formulations: Impact of surface treatments on particle toxicity. Toxicol Sci 2005;88(2):514-24.

11. Warheit DB, Hansen JF, Yuen IS, Kelly DP, Snajdr SI, Hartsky MA. Inhalation of high concentrations of low toxicity dusts in rats results in impaired pulmonary clearance mechanisms and persistent inflammation. Toxicol Appl Pharmacol 1997;145(1):10-22.

12. Nemmar A, Hoet PH, Vanquickenborne B, Dinsdale D, Thomeer M, Hoylaerts MF, et al. Passage of inhaled particles into the blood circulation in humans. Circulation 2002;105(4):411-4.

13. Nemmar A, Vanbilloen H, Hoylaerts MF, Hoet PH, Verbruggen A, Nemery B. Passage of intratracheally instilled ultrafine particles from the lung into the systemic circulation in hamster. Am J Respir Crit Care Med 2001;164(9):1665-8.

14. Driscoll KE, Costa DL, Hatch G, Henderson R, Oberdorster G, Salem $\mathrm{H}$, et al. Intratracheal instillation as an exposure technique for the evaluation of respiratory tract toxicity: Uses and limitations. Toxicol Sci 2000;55(1):24-35.

15. Leong BK, Coombs JK, Sabaitis CP, Rop DA, Aaron CS. Quantitative morphometric analysis of pulmonary deposition of aerosol particles inhaled via intratracheal nebulization, intratracheal instillation or noseonly inhalation in rats. J Appl Toxicol 1998;18(2):149-60.

16. Lam CW, James JT, Latch JN. Pulmonary toxicity of simulated lunar and Martian dusts in mice: II. Biomarkers of acute responses after intratracheal instillation. Inhal Toxicol 2002;14(9):917-28.

17. Lam CW, James JT, McCluskey R, Hunter RL. Pulmonary toxicity of single-wall carbon nanotubes in mice 7 and 90 days after intratracheal instillation. Toxicol Sci 2004;77(1):126-34.

18. Warheit DB, Webb TR, Reed KL, Frerichs S, Sayes CM. Pulmonary toxicity study in rats with three forms of ultrafine-TiO2 particles: Differential responses related to surface properties. Toxicology 2007;230(1):90-104

19. Reddy AR, Reddy YN, Krishna DR, Himabindu V. Pulmonary toxicity assessment of multiwalled carbon nanotubes in rats following intratracheal instillation. Environ Toxicol 2012;27(4):211-9.

20. Warheit DB, Laurence BR, Reed KL, Roach DH, Reynolds GA, Webb TR. Comparative pulmonary toxicity assessment of single-wall 
carbon nanotubes in rats. Toxicol Sci 2004;77(1):117-25.

21. Hamilton RF Jr, Wu Z, Mitra S, Shaw PK, Holian A. Effect of MWCNT size, carboxylation, and purification on in vitro and in vivo toxicity, inflammation and lung pathology. Part Fibre Toxicol 2013;10(1):57.

22. Berry JP, Arnoux B, Stanislas G, Galle P, Chretien J. A microanalytic study of particles transport across the alveoli: Role of blood platelets. Biomedicine 1977;27(9-10):354-7.

23. Brown JS, Zeman KL, Bennett WD. Ultrafine particle deposition and clearance in the healthy and obstructed lung. Am J Respir Crit Care Med 2002;166(9):1240-7.

24. Pekkanen J, Peters A, Hoek G, Tiittanen P, Brunekreef B, de Hartog J, et al. Particulate air pollution and risk of ST-segment depression during repeated submaximal exercise tests among subjects with coronary heart disease: The exposure and risk assessment for fine and ultrafine particles in ambient air (ULTRA) study. Circulation 2002;106(8):933-8.

25. Mercer RR, Scabilloni JF, Hubbs AF, Battelli LA, McKinney W, Friend S, et al. Distribution and fibrotic response following inhalation exposure to multi-walled carbon nanotubes. Part Fibre Toxicol 2013;10:33.

26. Mercer RR, Scabilloni JF, Hubbs AF, Wang L, Battelli LA, McKinney W, et al. Extrapulmonary transport of MWCNT following inhalation exposure. Part Fibre Toxicol 2013;10:38. 